\title{
Decisiones de inversión a través de opciones reales en el sector tecnológico ${ }^{1}$ \\ Investment decisions through real options in the technology sector
}

DOI: https://doi.org/10.17981/econcuc.40.1.2019.04

Artículo de investigación. Fecha de recepción: 09/11/2018 Fecha de aceptación: 01/03/2019

\author{
Armando Lenin Támara Ayús \\ Universidad EAFIT. (Medellín, Colombia) \\ atamaraa@eafit.edu.co \\ Susana Marín Arango \\ Universidad EAFIT. (Medellín, Colombia) \\ smarina@eafit.edu.co
}

\author{
Santiago Cadavid Álvarez \\ Universidad EAFIT. (Medellín, Colombia) \\ scadavi6@eafit.edu.co
}

Para citar este artículo:

Támara, A., Marín, S. y Cadavid, S. (2019). Decisiones de inversión a través de opciones reales en el sector tecnológico. Económicas CUC, 40(1). 61-75. DOI: http://doi.org/10.17981/econcuc.40.1.2019.04

Resumen

El sector de tecnología se caracteriza por la existencia de proyectos con flexibilidad, en gran medida por la oportunidad de mejorar el producto antes de ser enviado al mercado, como es el caso de los video juegos. La teoría bajo la cual se estudian y analizan este tipo de proyectos son las opciones reales, las cuales complementan las metodologías tradicionales, como el valor presente neto y la tasa interna de retorno. La metodología aplicada en este trabajo se basa en la técnica de Árboles Binomiales, permitiendo analizar y valorar la opción de diferir en un tiempo previamente estipulado. El resultado de considerar la opción real aumenta el valor del proyecto en un $33 \%$, sobre su valor calculado a través del valor presente neto, a la vez, reafirmando la importancia de tener en cuenta la opción real a la hora de valorar proyectos del sector tecnológico. Se concluye que la creación de un proyecto de video juegos, presenta alta aplicabilidad en los conceptos de opciones reales, debido a los componentes de incertidumbre que suponen este tipo de decisiones de inversión.

Palabras clave: Opciones reales; opciones de diferir; sector tecnología.

\begin{abstract}
The technology sector is characterized by the existence of projects with flexibility, largely by the opportunity to improve the product before being sent to market, as is the case of video games. The theory under which this type of projects are studied and analyzed are the real options, which complement the traditional methodologies, such as the net present value and the internal rate of return. The methodology applied in this work is based on the Binomial Trees technique, allowing to analyze and value the option to differ in a previously stipulated time. The result of considering the real option increases the value of the project in $33 \%$, on its calculated value through the net present value, at the same time, reaffirming the importance of taking into account the real option at the time of valuing projects of the technological sector. It is concluded that the creation of a video game project has high applicability in the concepts of real options, due to the uncertainty components that this type of investment decisions suppose.
\end{abstract}

Keywords: Real options; options to differ; technology sector.

\footnotetext{
${ }^{1}$ Este artículo es resultado de investigación enmarcado dentro del Grupo de Investigación en Finanzas y Banca (Gifyb) en el pregrado de Finanzas de la Escuela de Economía y Finanzas de la Universidad EAFIT (Colombia).
} 


\section{INTRODUCCIÓN}

Teniendo en cuenta la relevancia que tiene el sector de tecnología para el crecimiento económico de cualquier país y los grandes avances en este sector, sucedidos en los últimos años, las inversiones en este aspecto brindan un claro ejemplo de los beneficios y diferencias existentes entre las distintas técnicas utilizadas en la valoración financiera de proyectos y la metodología de las opciones reales. En este trabajo se implementa la teoría de las opciones reales dentro del marco de la valoración de proyectos relacionados al sector de la tecnología, más específicamente los referentes a la creación de video juegos, lo cual resulta ampliamente relevante. Teniendo en cuenta que durante el año 2017 este subsector se posicionó como el de mayor facturación de la industria digital. Y es que la industria de video juegos, la cual lleva poco más de medio siglo de desarrollo, anualmente genera cerca de tres veces más ventas a nivel global que la misma industria cinematográfica, según Rohner (2018), en 2016 la industria de video juegos generó ventas agregadas a nivel global por valor de US $\$ 106.000$ millones aproximadamente.

En el presente estudio y análisis presentamos, como primero un referente a las técnicas más comunes en la valuación de proyectos, seguidamente, se encuentra una sección relacionada a la teoría de opciones reales, posteriormente, sigue una sección de revisión de literatura en torno a las opciones reales en el sector tecnológico y finalizamos, con la aplicación de un caso y las conclusiones.

\section{Técnicas de valuación de proyectos}

Tradicionalmente la manera de evaluar la viabilidad financiera de un proyecto se ha centrado en el cálculo y análisis de los flujos de caja generados producto de una inversión inicial, desde lo cual se han aplicado diferentes metodologías de valoración, siendo tres los más destacados, que para el caso son: valor presente neto (VPN), tasa interna de retorno (TIR) y período de recuperación (PR), haciendo énfasis en que este último lo pueden trabajar como período de recuperación descontado (PRD).

El VPN es la sumatoria de todos los flujos esperados del proyecto descontados hasta el periodo inicial, donde se considera, además, la inversión inicial realizada. Estos flujos son descontados a una tasa consistente con el costo de la estructura de capital, además, de ser sensibilizada con mediciones del sector, la cual, adicionalmente, debe medir correctamente el costo de oportunidad y el riesgo de la inversión. Sin embargo, existe una discrepancia alrededor del supuesto de que la tasa de descuento se mantenga igual durante todo el período de evaluación, atendiendo a que la estructura financiera puede variar periodo a periodo. Las reglas de valoración tradicionales nos sugieren que todo proyecto con VPN positivo deberá ser aceptado, y aquel con VPN negativo deberá ser rechazado. Aunque estas reglas parecen por sí solas un poco estáticas, debemos recordar que también deben tenerse en cuenta conceptos como la capacidad de generación de sinergias y demás beneficios generados para la organización.

En relación a la TIR, se considera como la tasa que hace que el VPN sea cero y refleja la tasa de rendimiento promedio 
de la inversión. Según las reglas tradicionales, el criterio de la TIR entra a ser un elemento diferente según cada tipo de inversionista, según su rentabilidad esperada y nivel de riesgo deseado. Según esto, deberá rechazarse el proyecto cuando este entregue una rentabilidad por debajo de la Tasa Mínima de Retorno exigida por el inversionista. Por otra parte, el PR es una métrica del tiempo en relación a que indica el momento en el cual se recupera la inversión realizada. Al igual que la TIR, la aceptación o no del proyecto según este criterio, dependerá de las preferencias personales del inversionista, debido a que puede rechazar proyectos, cuyo tiempo de recuperación esté por encima del esperado por los inversionistas.

Aunque la aplicación de estas reglas se ha mantenido durante un largo tiempo, se han encontrado diversos estudios que demuestran que estas metodologías tradicionales representan grandes obstáculos para la implementación de innovaciones empresariales, ya que, bajo estos supuestos de valoración, la decisión de inversión (con la valoración resultante) solo se mantiene si esta es tomada en el corto plazo. Adicionalmente, estas pueden presentar un sesgo importante, al tratar únicamente como elementos fundamentales para la toma de decisiones los flujos de caja del proyecto, ya que, en ocasiones, pueden subvalorar las ganancias relacionadas con la ejecución de este.

\section{Teoría de las opciones reales}

Los diferentes estudios relacionados con los métodos tradicionales de valoración de proyectos han mostrados que estas técnicas presentan algunas limitaciones $\mathrm{y}$ dificultades a la hora de ser incorporados a entornos variables o escenarios que muestren algún tipo de flexibilidad, tal y como lo plantea Calle y Tamayo (2009). En contraste, la teoría de opciones reales soluciona el problema de la flexibilidad operativa existente en los diferentes proyectos de inversión que se planten en el presente, a la vez, dicha flexibilidad es considerada como un factor determinante en la toma de decisión referente a la ejecución o no del proyecto. Bajo esta mirada, fue Myers (1977) quien estableció el término de opciones reales a los diferentes trabajos que incluyen más de una alternativa de desarrollo y, por ende, trabajan el concepto teórico de las opciones reales en lo referente a la valoración de activos no financieros, tomando como referencia la flexibilidad, en las inversiones en activos reales tales como la inversión en investigación, tecnología, construcción, explotación de minas y expansión de plantas de manufactura, entre otros.

En el marco de las opciones financieras, Mascareñas (2004) ha definido a las opciones como el derecho que posee una persona o institución entorno a la realización de una operación de terminada en un período de tiempo estipulado, sin embargo, se especifica que no existe la obligación por parte del poseedor de ejecutar la operación. Bajo esta aclaración, las opciones se pueden clasificar de acuerdo a si son opciones de compra (Call) o si son opciones de ventas (Put), las primeras se consideran opciones tipo compra y las segundas son opciones tipo venta. Por otra parte, según la posibilidad que se tengan para ejercer las opciones, estas se pueden clasificar en americanas o europeas, es así como, Calle y Tamayo (2009) estipulan que el poder de ejercerse en cualquier momento dentro del período estipulado de la opción, son propias solo en las opciones tipo americanas, mientras que, las 
opciones europeas estipulan que solo se pueden ejercer en el momento en que el tiempo estipulado llegue a su fin.

Para el cálculo de las opciones se han desarrollado tres metodologías, la primera, es el modelo Black Scholes, el cual se establece bajo el supuesto de que el precio de las acciones persigue un curso aleatorio, es decir, que para un período corto de tiempo sigue una distribución normal. La segunda, la Simulación Monte Carlo, que consiste en tomar diferentes fuentes de incertidumbre presentes en la opción y simularlas. La tercera, son los Árboles Binomiales propuesta por Cox, Ross y Rubinstein en 1974, la cual está basada en un modelo discreto que plantea dos valores posibles bajo un escenario positivo y otro negativo, donde cada uno de ellos posee una probabilidad de ocurrencia.

En la actualidad existen diferentes tipos de opciones reales, sin embargo, las opciones más comunes que se presentan en los diferentes proyectos son las opciones de crecimiento, las cuales otorgan el derecho de ampliar el proyecto aumentando el tamaño de la producción existente o incursionando en nuevos mercados. Señalamos también las opciones de protección o abandono, las cuales conceden el derecho a reducir o cerrar operaciones en el futuro, y las opciones de aprendizaje, que dan la posibilidad de aplazar la decisión de incursionar en un nuevo mercado para que en él puedan contar con mayor información.

Mascareñas (2008) indica que, dentro del desarrollo de la valoración financiera de un proyecto de inversión, se debe calcular el valor del proyecto sin considerar ninguna opción, utilizando las técnicas tradicionales, especialmente el VPN, y luego calcular el valor de la o las opciones presentes en el proyecto, de tal manera que, el valor total del proyecto será el VPN más el valor de las opciones. Basado en lo anterior, Calle y Tamayo (2009) han mostrado como el valor de los proyectos que poseen una o más opciones reales, han incrementado dicho valor, es así como, proyectos donde se identifique cualquier tipo de flexibilidad se hará necesario la aplicación de la teoría de opciones reales y por ende, el reconocimiento dentro del valor total del proyecto, el valor de la o las opciones reales. Por otra parte, Mascareñas (2008) ya había indicado que el uso de la teoría de opciones reales ha enriquecido el entendimiento en la formulación y articulación entorno a las decisiones sobre la aceptación o negación de realizar cualquier proyecto de inversión. Sin embargo, Calle y Tamayo (2009) mencionan la importancia de contar con una adecuada valoración del proyecto, ya que, el éxito de la valoración dependerá de la adecuada construcción de los supuestos e información disponible para realizarla, a lo que, Mascareñas (2008) indica que la integración del presupuesto de capital y la planificación estratégica, solo es posible a través de las opciones reales, a la vez que, ofrece un método sólido de análisis dado que involucra tanto decisiones financieras como reales.

\section{Opciones reales en el sector}

de telecomunicaciones

En el sector tecnológico, Martínez y Aizenstat (2006) realizaron uno de los primeros estudios acerca de la aplicación de las opciones reales sobre la toma de decisiones empresariales. En el presente trabajo, presentamos diferentes métodos y análisis del entorno en el cual se encontraban las empresas mexicanas, que re- 
currieron al uso de estas, durante el año 2006 , adicionalmente, realizaron diferentes cálculos para medir la flexibilidad en los proyectos, encontrando que la metodología de opciones reales también puede ser aplicada a los procesos de fusiones y adquisiciones. Por otra parte, González (2008) indica que aquellas organizaciones cuya capacidad de innovar son altas y que además poseen tecnologías blandas y duras, son las que finalmente tienen las mayores posibilidades de crecer y mantenerse en los mercados globales y con altos niveles de competencia.

En el caso de Cassimon, Engelen y Yordanov (2011) establecieron que la volatilidad presente en la valoración de proyectos relacionados con software, tiende a presentar diferentes variaciones en referencia a la fase en que se encuentre el proyecto, debido en gran parte a la incertidumbre presente en el éxito de su aplicación. Blazenko, Pavlov y Eddy-Sumeke (2012) realizan una comparación sobre las decisiones de inversión en tecnología entre start-ups antes de su comercialización, y firmas que actualmente se encuentran en operación después de esta. Los hallazgos se sintetizan en afirmar que las compañías que actualmente se encuentran en operación, utilizan y enfocan sus esfuerzos en investigación y desarrollo, para mejorar el nivel de ingresos actuales, mientras que las Start-ups lo hacen con el fin de mejorar la perspectiva de ingresos a futuro, además, que la inversión en I+D adquiere mayor importancia a medida que el crecimiento se acelera.

Della, Gryglewicz y Kort (2012) muestran una relación en cuanto a las implicaciones de las decisiones de inversión en tecnología, afirmando, que estas decisiones son más difíciles y más demandantes financieramente, mientras menos acelerada sea la curva de aprendizaje relacionada con la incorporación de esta. Franklin y Diallo (2012) proponen un modelo y una metodología para valorar la opción de retrasar las decisiones de inversión de red teniendo en cuenta la demanda y las incertidumbres tecnológicas en las redes de telecomunicaciones, además, identifican que diferentes elementos de red están sujetos a diferentes incertidumbres tecnológicas y de demanda, por lo que, se calcula un factor de marcado para cada elemento de red principal. Por otra parte, Trigeorgis y Ioulianou (2013) modelaron las oportunidades de crecimiento de la compañía EchoStar utilizando como activo subyacente para las opciones de expansión, el flujo de efectivo descontado 'base' (DCF), después de eliminar la tasa de crecimiento constante en el supuesto de DCF de valor terminal.

Wadhwa y Basu (2013) desarrollan un enfoque de opciones reales en los negocios Venture Capital y sugieren que la teoría de opciones reales proporciona insumos sobre cómo los gerentes en la empresa en riesgo enfrentan y mitigan la incertidumbre relacionada con la tecnología y el mercado en este tipo de negocios. Adicionalmente, sostienen que desde la teoría de opciones reales una opción de bajo costo que preserve el acceso a inversiones estratégicas futuras es atractiva en condiciones de incertidumbre, crear una opción, en lugar de hacer compromisos de recursos más irreversibles y extensos, reduce el posible efecto negativo en una empresa cuando no se acumulan oportunidades estratégicas valiosas. Por otra parte, Villani (2014) propone una metodología en opciones reales para valorar la oportunidad de inversión en I + D de una empresa líder en el mercado, incorporando una dimensión 
competitiva, es decir, asumiendo que el pionero (líder) puede perder los "dividendos competitivos" si la opción real no se ejerce.

Más recientemente, los estudios de Wesseh y Lin (2016) consideraron la flexibilidad sobre el despliegue de la tecnología en respuesta al costo de energía estocástica no renovable, para evaluar la viabilidad de los proyectos de energía eólica en China utilizando un tipo de opción real de retener. Finalmente, Leippold y Stromberg (2017) plantean que en la decisión de invertir en proyectos empresariales implementando tecnología disponible o diferir hasta que esta sea más eficiente, a lo que se debe considerar tres aspectos: la probabilidad de una nueva tecnología, la entrada de otros individuos y el impacto en la rentabilidad, relacionado con los riesgos no diversificables relacionados al proyecto, por lo que, concluyen que el impacto del riesgo no diversificable en el tiempo de las opciones es ambiguo y depende de la frecuencia del cambio tecnológico.

\section{Aplicación}

Como caso de estudio se seleccionó la valoración de un proyecto cuyo objetivo es la creación de un video juego, cuya inversión es de $\$ 50$ millones por concepto de diseño e implementación. El mantenimiento anual del aplicativo tiene un valor de $\$ 10$ millones y el costo anual de la plataforma es de $\$ 2$ millones. El precio de descarga se plantea en $\$ 8.950$ y se proyecta unas 4.000 descargas por año, adicionalmente, se espera una tasa de crecimiento anual en el precio de la descarga del $5 \%$. La financiación del proyecto es de $50 \%$ deuda y $50 \%$ capital propio, la deuda tiene un costo del $18 \%$
EA con amortizaciones iguales para un período de 5 años a una tasa impositiva del 33\%. La tasa mínima requerida por los accionistas es del $30 \%$ y se plantea diferir la decisión de inversión por un tiempo de 5 años con revisión cada año, dicha espera produce una pérdida del $15 \%$ sobre las ganancias por año. A la fecha, la tasa libre de riesgo tomada con base en los TES tipo B son del 6,7\% EA.

El primer paso para la realización de la evaluación financiera del proyecto, fue calcular el costo de la estructura financiera de este, para lo cual se tomó como el promedio ponderado del capital, más conocido como el WACC (Weighted Average Cost of Capital), así:

$\mathrm{WACC}=\mathrm{Kd} \cdot \% \mathrm{D} \cdot(1-\mathrm{T})+\mathrm{Ke} \cdot \% \mathrm{E}$
$\mathrm{WACC}=18 \% \cdot 50 \% \cdot(1-33 \%)+30 \% \cdot 50 \%$
$\mathrm{WACC}=21 \%$

Donde:

$K_{d}$ : Costo de la deuda.

$K_{\mathrm{e}}$ : Costo del patrimonio.

$T$ : Tasa impositiva.

$\% D$ : Participación de la deuda calculada como Deuda/ (Deuda + Patrimonio).

$\% E$ : Participación del patrimonio calculado como Patrimonio/ (Deuda + Patrimonio).

Basados en la formula (1), el proyecto posee un costo de su estructura financiera equivalente al $21 \%$ EA, esta tasa será utilizada para descontar los diferentes flujos de cajas que se originen producto de la inversión realizada. Por lo tanto, el segundo paso es calcular el flujo de caja libre para los diferentes períodos establecidos en el proyecto y con base en estos encontrar el VPN y la TIR, los resultados se muestran en la tabla 1. 
TABLA 1

Calculo del VPN y TIR

\begin{tabular}{|c|c|c|c|c|c|c|}
\hline Años & 0 & 1 & 2 & 3 & 4 & 5 \\
\hline $\begin{array}{l}\text { Utilidad } \\
\text { Operativa }\end{array}$ & & $\$ 15.590 .000$ & $\$ 16.869 .500$ & $\$ 18.212 .975$ & $\$ 19.623 .624$ & $\$ 21.104 .805$ \\
\hline Amortización & & $\$ 10.000 .000$ & $\$ 10.000 .000$ & $\$ 10.000 .000$ & $\$ 10.000 .000$ & $\$ 10.000 .000$ \\
\hline Inversiones & $\$ 50.000 .000$ & & & & & \\
\hline $\begin{array}{c}\text { Flujo de Caja } \\
\text { Operativo }\end{array}$ & $-\$ 50.000 .000$ & $\$ 25.590 .000$ & $\$ 26.869 .500$ & $\$ 28.212 .975$ & $\$ 29.623 .624$ & $\$ 31.104 .805$ \\
\hline $\begin{array}{l}\text { Impuestos } \\
\text { operativos }\end{array}$ & $\$$ & $\$ 5.144 .700$ & $\$ 5.566 .935$ & $\$ 6.010 .282$ & $\$ 6.475 .796$ & $\$ 6.964 .586$ \\
\hline $\begin{array}{l}\text { Flujo de caja } \\
\text { libre }\end{array}$ & $-\$ 50.000 .000$ & $\$ 20.445 .300$ & $\$ 21.302 .565$ & $\$ 22.202 .693$ & $\$ 23.147 .828$ & $\$ 24.140 .219$ \\
\hline $\begin{array}{c}\text { Valor } \\
\text { Terminal }\end{array}$ & & & & & & $\$ 158.123 .707$ \\
\hline FCL VT & $-\$ 50.000 .000$ & $\$ 20.445 .300$ & $\$ 21.302 .565$ & $\$ 22.202 .693$ & $\$ 23.147 .828$ & $\$ 182.263 .927$ \\
\hline VPN & $\$ 74.930 .520$ & & & & & \\
\hline TIR & $57 \%$ & & & & & \\
\hline
\end{tabular}

Fuente: Elaboración propia.

TABLA 2

Escenarios de las variables de entrada para la simulación

\begin{tabular}{llll}
\hline \multicolumn{1}{c}{ Variable } & \multicolumn{3}{c}{ Escenarios } \\
& Pesimista & Moderado & Optimista \\
\hline Precio de descarga & $\$ 7.950$ & $\$ 8.950$ & $\$ 9.950$ \\
$\begin{array}{l}\text { Descargas por año } \\
\begin{array}{l}\text { Tasa de crecimiento del precio de la } \\
\text { descarga }\end{array}\end{array}$ & 3.000 & 4.000 & 5.000 \\
\hline
\end{tabular}

Fuente: Elaboración propia.

El VPN del proyecto es de $\$ 74.930 .520$, lo cual, teniendo en cuenta las reglas de decisión tradicionales de valoración de proyectos implicarían su realización por las posibilidades de generación de valor, adicionalmente, la Tasa Interna de Retorno (TIR) calculada es del $57,32 \%$, esta supera lo requerido por los accionistas, su estimación es del 30\%, lo que permite la viabilidad financiera del proyecto. Posteriormente, con el fin de obtener una métrica precisa de los riesgos inherentes al proyecto, se procedió a realizar una simulación con 10.000 intentos, para así cuantificar los efectos de variaciones en los precios y cantidades de descarga por año en los principales indicadores de valor para la toma de decisiones. La tabla 2 muestra los diferentes escenarios establecidos para la simulación, en el cual, bajo un escenario pesimista el precio inicial de descarga se ubicaría en $\$ 7.950$ con incrementos en este del $4 \%$, y bajo un escenario optimista, caso en el cual el precio inicial estaría en $\$ 9.950$ con aumentos del $6 \%$ anuales. 


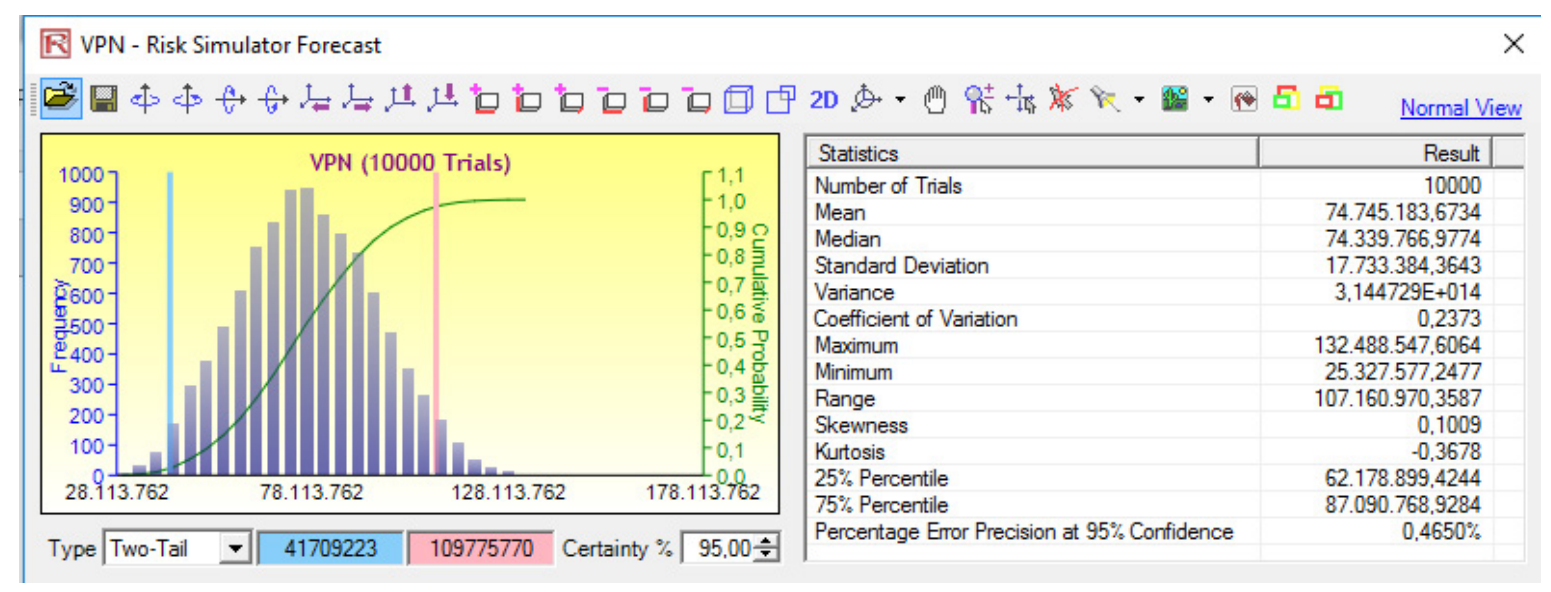

Figura 1. Simulación del VPN.

Fuente: Aalida del @Risk Simulator.

D. TIR - Risk Simulator Forecast

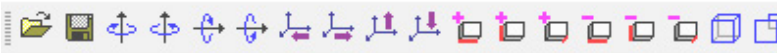

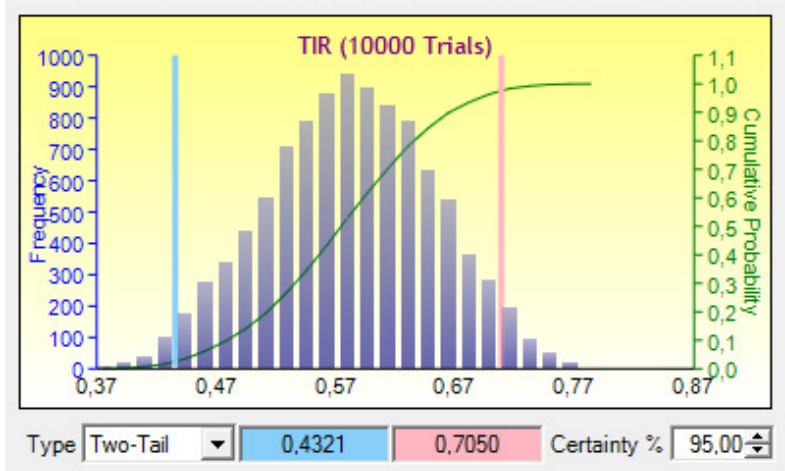

\begin{tabular}{|c|c|}
\hline Statistics & Result \\
\hline Number of Trials & 10000 \\
\hline Mean & 0,5702 \\
\hline Median & 0,5711 \\
\hline Standard Deviation & 0,0706 \\
\hline Variance & 0,0050 \\
\hline Coefficient of Variation & 0,1238 \\
\hline Maximum & 0,7795 \\
\hline Minimum & 0,3553 \\
\hline Range & 0.4242 \\
\hline Skewness & $-0,0596$ \\
\hline Kurtosis & $-0,3716$ \\
\hline $25 \%$ Percentile & 0,5213 \\
\hline $75 \%$ Percentile & 0.6203 \\
\hline Percentage Error Precision at $95 \%$ Confidence & $0,2426 \%$ \\
\hline
\end{tabular}

Figura 2. Simulación de la TIR.

Fuente: Salida del @Risk Simulator.

Teniendo en cuenta las características del proyecto, y las variables mencionadas anteriormente para la simulación, podemos observar en la figura 1 que el valor medio esperado del VPN es de $\$ 74.745 .183$, por lo tanto, de igual manera, implicaría la aceptación de este. También, se observa que, en el rango observado, el valor mínimo y máximo obtenido en la simulación representan valores positivos, es decir, considerando únicamente esta regla y la volatilidad asociada a los flujos de caja presentados en cualquiera de los escenarios posibles, el proyecto resultaría viable, aún, con un precio inicial de descarga inferior al inicialmente considerado.

En lo concerniente a la Tasa Interna de Retorno (TIR), se procedió a realizar el mismo proceso de simulación, aquí se observa que bajo los escenarios más adversos la TIR se encuentra en niveles aceptables (35,53\%), superando la Tasa Mínima de Retorno (TMR) requerida por los inversionistas. Por lo tanto, aún bajo estos escenarios, el proyecto sería aceptable, con una media de $57,02 \%$ y logrando niveles máximos de $77,95 \%$, tal y como lo muestra la figura 2. 


$$
\operatorname{Ln}\left(\frac{\mathrm{VP}_{1}}{\mathrm{VP}_{0}}\right)=\operatorname{Ln} \frac{- \text { Inversión }+\mathrm{VP}_{1}\left(\mathrm{FC}_{1}\right)+\mathrm{VP}_{2}\left(\mathrm{FC}_{2}\right)+\cdots+\mathrm{VP}_{\mathrm{t}}\left(\mathrm{FC}_{\mathrm{t}}\right)}{\mathrm{VP}_{1}\left(\mathrm{FC}_{1}\right)+\mathrm{VP}_{2}\left(\mathrm{FC}_{2}\right)+\cdots+\mathrm{VP}_{\mathrm{t}}\left(\mathrm{FC}_{\mathrm{t}}\right)}
$$

En esta parte de la valoración financiera del proyecto se procedió a calcular la volatilidad con base en los flujos de caja libre, implementado la formula 2.

La salida que arroja el @Risk Simulator la cual se puede ver en la figura 3, muestra una desviación estándar de 0,1443 , equivalente a una volatilidad del $14,43 \%$. Lo anterior es consecuente con los cambios establecidos en el nivel de precios utilizado en la simulación.

Con el objeto de determinar la sensibilidad estadística de las variables con respecto a otras en el proyecto de video juegos, se procedió a realizar un análisis de tornado, logrando jerarquizar las variables de acuerdo, al efecto que posean por sobre los resultados obtenidos tanto en el VPN como en la volatilidad. Adicionalmente, este despliegue de jerarquía relativa de una variable de entrada versus otra, compara los efectos de todas las variables de entrada sobre los resultados obtenidos, detectando en este caso que las dos variables más significativas en la jerarquía bajo este análisis son la inversión inicial y el VPN, como se puede ver en la figura 4.

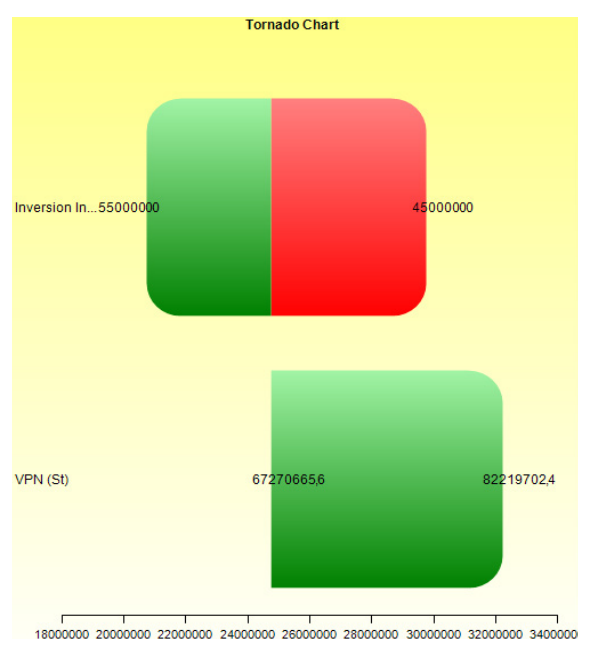

Figura 4. Análisis de tornado Fuente: salida del @Risk Simulator.

Cabe anotar, que, para las dos variables detectadas, la longitud de la barra indica la cantidad de cambio que la inversión inicial y el VPN causan por sobre los resultados. Estos resultados van alineados con la realidad de este mercado, dado que la inversión inicial que se deben hacer en este tipo de proyectos es muy grande, y esto a su vez, se relaciona con el valor presente del proyecto, el cual es decisivo a la hora de tomar la decisión de inversión.

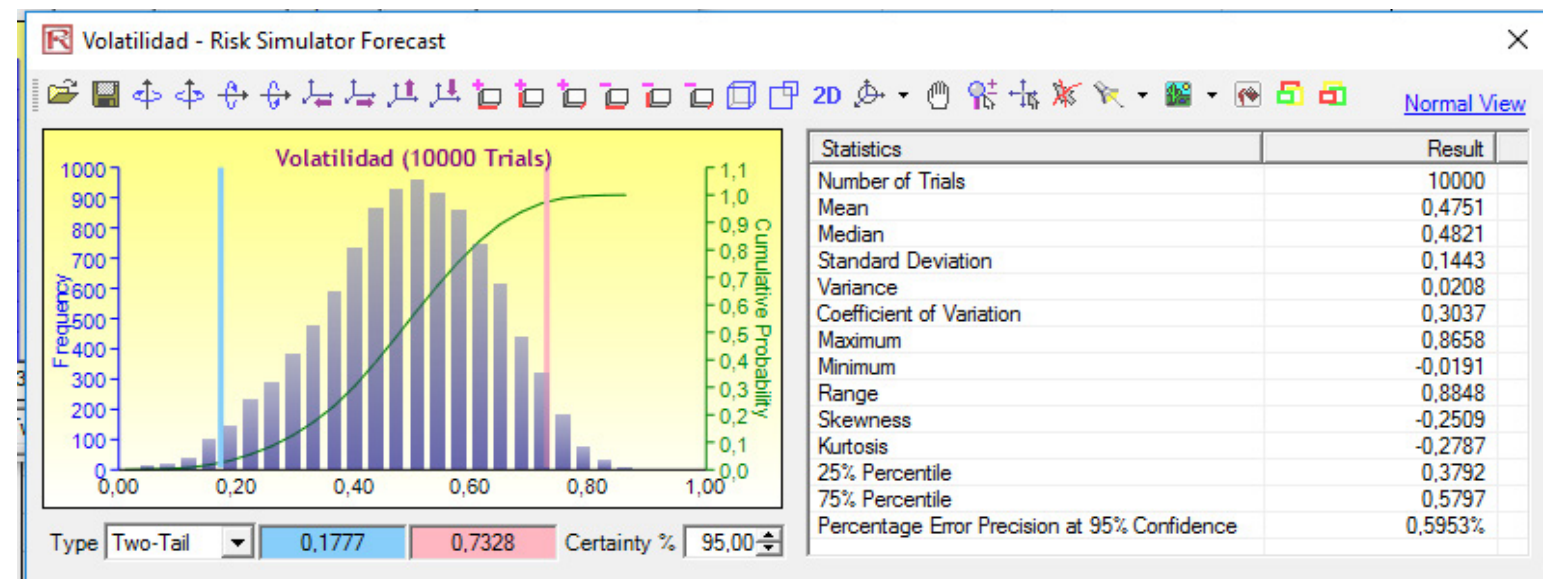

Figura 3. Calculo de la volatilidad.

Fuente: Salida del @Risk Simulator. 


\section{Valorando la Opción Real}

El proyecto analizado posee la opción de diferir la inversión en una ventana de tiempo de 5 años, con la posibilidad de hacer una valuación al final de cada año, sin embargo, como consecuencia de esta flexibilidad en la decisión de espera, el efecto esperado sobre los flujos de caja, arroja una pérdida del $15 \%$ sobre las ganancias por año. Para el cálculo del valor de la opción a diferir se utiliza la técnica de Árboles binomiales desarrollada por Copeland y Antikarov (2003), elaborando el árbol del subyacente, con base en los parámetros: tiempo en el que perdura la opción $(T)$, tiempo de revisión de la opción $(\Delta t)$, número de momentos que posee el árbol (n), expansión del árbol del subyacente $(u)$, contracción del árbol del subyacente $(d)$ y volatilidad de los flujos de caja Establecidos los parámetros requeridos para elaborar el árbol del subyacente, se realizan los cálculos necesarios, iniciando con el número de momentos que debe tener el árbol (3).

$$
n=\frac{T}{\Delta t}=\frac{5}{1}=5 \text { momentos }
$$

Seguidamente, se calculan los parámetros de expansión y contracción, así:

$$
\begin{aligned}
u=e^{\sigma^{*} \sqrt{ } \Delta t}=e^{0.1443^{*} \sqrt{ }{ }^{1}} & =1,16 \\
d=\frac{1}{u}=\frac{1}{1,16} & =8,7
\end{aligned}
$$

Encontrados los parámetros $u$ y $d$, se elabora el árbol del subyacente, iniciando con el VPN medio obtenido a través de la simulación desarrollada a las técnicas tradicionales vista en la sección anterior. De tal manera que, el Árbol binomial del subyacente se elabora multiplicando el VPN medio por $u$, encontrando así el mejor escenario en el momento $1 \mathrm{y}$, multiplicando por d para encontrar el peor escenario en el mismo momento 1. Por lo tanto, en referencia a estos dos escenarios se calcularán las demás ramas pertenecientes a los demás escenarios para los restantes momentos, aplicando la misma metodología que se desarrolló para el momento 1, obteniendo así el árbol del subyacente que se presenta en la figura 3.

\begin{tabular}{|c|c|c|c|c|c|}
\hline 0 & 1 & 2 & 3 & 4 & 5 \\
\hline & & & & & $\$ 68.237 .218$ \\
\hline & & & & $\$ 69.491 .821$ & \\
\hline & & & $\$ 70.769 .491$ & & $\$ 51.130 .957$ \\
\hline & & $\$ 72.070 .653$ & & $\$ 52.071 .046$ & \\
\hline & $\$ 73.395 .737$ & & $\$ 53.028 .420$ & & $\$ 38.313 .033$ \\
\hline \multirow[t]{6}{*}{$\$ 74.745 .184$} & & $\$ 54.003 .395$ & & $\$ 39.017 .453$ & \\
\hline & $\$ 54.996 .297$ & & $\$ 39.734 .824$ & & $\$ 28.708 .411$ \\
\hline & & $\$ 40.465 .385$ & & $\$ 29.236 .241$ & \\
\hline & & & $\$ 29.773 .776$ & & $\$ 21.511 .553$ \\
\hline & & & & $\$ 21.907 .062$ & \\
\hline & & & & & $\$ 16.118 .862$ \\
\hline
\end{tabular}

TABla 3

Árbol del subyacente 
Para elaborar el árbol del ejercicio que se muestra en la tabla 4, se debe hacer la equivalencia de la opción real frente a la opción financiera, en este caso, la opción de diferir equivale a una opción Call. Por lo tanto, el cálculo de las diferentes ramas del árbol para cada momento se calcula de la siguiente manera:

$$
\begin{aligned}
& \text { Max ((VPNt-precio de ejercicio); } \\
& \text { Max }((V P N t-\text { Costo de la inversión }) ; 0) \\
& \operatorname{Max}((V P N t-\$ 50.000 .000) ;
\end{aligned}
$$

Elaborado el árbol del ejercicio se procede a realizar el árbol de la opción "viva", para lo cual, inicialmente se toma el valor de la opción en el último período y con base en estos valores comenzamos a descontar los períodos de derecha a izquierda bajo el mismo procedimiento que se realizó en los árboles anteriores aplicando la siguiente fórmula (7):
El parámetro q es la probabilidad neutral al riesgo y $r_{f}$ es la tasa libre de riesgo (tasa efectiva de los TES tipo $B$ publicada por el Banco de la República), la cual se calcula de la siguiente manera:

$$
\begin{aligned}
& q=\frac{a-d}{u-d} \\
& a=e^{r f^{*} \Delta T} \\
& q=\frac{1,07-0,87}{1,16-0,87}=1,07
\end{aligned}
$$

Teniendo en cuenta el desarrollo anterior, se presenta el árbol de la opción "viva" en la tabla 5.

Tomando como referencia los tres árboles mostrados anteriormente, se procede a la elaboración del árbol de la opción (tabla 6) aplicando la formula 10.

\begin{tabular}{|c|c|c|c|c|c|}
\hline 0 & 1 & 2 & 3 & 4 & 5 \\
\hline & & & & & $\$ 18.237 .218$ \\
\hline & & & & $\$ 19.491 .821$ & \\
\hline & & & $\$ 20.769 .491$ & & $\$ 1.130 .957$ \\
\hline & & $\$ 22.070 .653$ & & $\$ 2.071 .046$ & \\
\hline & $\$ 23.395 .737$ & & $\$ 3.028 .420$ & & $\$-$ \\
\hline \multirow[t]{6}{*}{$\$ 24.745 .184$} & & $\$ 4.003 .395$ & & $\$-$ & \\
\hline & $\$ 4.996 .297$ & & $\$-$ & & $\$$ - \\
\hline & & $\$-$ & & $\$-$ & \\
\hline & & & $\$-$ & & $\$$ - \\
\hline & & & & $\$-$ & \\
\hline & & & & & $\$-$ \\
\hline
\end{tabular}

Max (arbol de la opcion viva; arbol del ejercicio) (10)

$$
\frac{(\text { Max }(\text { alza } 5 \text { de la OV; laza } 5 \text { del } E) * q+\text { Max (baja } 5 \text { de la OV;baja } 5 \text { del } E) *(1-q)}{(1+r f)^{\Delta T}}
$$

TABLA 4

Árbol del ejercicio

Fuente: Elaboración propia. 
TABla 5

Árbol de la opción "viva"

\begin{tabular}{|c|c|c|c|c|c|}
\hline $\mathbf{0}$ & 1 & 2 & 3 & 4 & 5 \\
\hline & & & & & $\$ 18.237 .218$ \\
\hline & & & \multicolumn{3}{|c|}{$\$ 12.272 .340$} \\
\hline & & & $\$ 13.360 .607$ & & $\$ 1.130 .957$ \\
\hline & & $\$ 14.468 .883$ & & $\$ 741.207$ & \\
\hline & $\$ 15.597 .536$ & & $\$ 1.357 .322$ & & $\$-$ \\
\hline \multirow[t]{5}{*}{$\$ 16.746 .940$} & & $\$ 1.984 .765$ & & $\$-$ & \\
\hline & $\$ 2.623 .744$ & & $\$-$ & & $\$-$ \\
\hline & & $\$-$ & & $\$-$ & \\
\hline & & & $\$-$ & & $\$-$ \\
\hline & & & & $\$-$ & \\
\hline
\end{tabular}

Fuente: Elaboración propia.

TABLA 6

Árbol de la opción

\begin{tabular}{|c|c|c|c|c|c|}
\hline 0 & 1 & 2 & 3 & 4 & 5 \\
\hline & & & & & $\$ 18.237 .218$ \\
\hline & & & & $\$ 19.491 .821$ & \\
\hline & & & $\$ 20.769 .491$ & & $\$ 1.130 .957$ \\
\hline & & $\$ 22.070 .653$ & & $\$ 2.071 .046$ & \\
\hline & $\$ 23.395 .737$ & & $\$ 3.028 .420$ & & $\$-$ \\
\hline \multirow[t]{6}{*}{$\$ 24.745 .184$} & & $\$ 4.003 .395$ & & $\$-$ & \\
\hline & $\$ 4.996 .297$ & & $\$-$ & & $\$-$ \\
\hline & & $\$-$ & & $\$-$ & \\
\hline & & & $\$-$ & & $\$-$ \\
\hline & & & & $\$-$ & \\
\hline & & & & & $\$-$ \\
\hline
\end{tabular}

Fuente: Elaboración propia.

Como hemos mencionado anteriormente, la nueva corriente de valoración de opciones reales nos sugiere que el valor total del proyecto está compuesto por la sumatoria del VPN de los flujos de caja desconectados y el valor de la opción real.
Bajo este concepto, obtenemos un Valor total del proyecto de $\$ 99.861 .041$, compuesto por un VPN de $\$ 74.930 .520$, y una opción real de $\$ 24.745 .184$. Como puede observarse, según los criterios básicos de valoración de proyectos este hubiera sido 
aceptado ya que supone un VPN positivo, pero si desconocemos el valor de la opción real estaríamos dejando de percibir este valor, lo cual, nos indica que, al añadir los componentes de flexibilidad, el proyecto tiene mayor capacidad de generación de valor.

\section{Conclusiones}

El uso de las opciones reales como técnica o modelo cuantitativo y cualitativo es crucial en la valoración de proyectos en el sector de telecomunicaciones, dada las situaciones de incertidumbre que presenta este sector. Esta metodología ha adquirido protagonismo reciente con el mayor nivel de proyectos asociados a este sector, donde, como ya señalamos anteriormente, los estudios han estado centrados en observar las consecuencias de las inversiones en tecnología (cuantificadas ocasionalmente como investigación y desarrollo), de acuerdo con las etapas y condiciones asociadas a cada uno de los proyectos, y a la conveniencia de realizar estos.

La tendencia a incluir las opciones reales dentro de la valoración de proyectos es una tendencia relativamente nueva, asociada a las mayores posibilidades de conexión entre los diferentes mercados, y a la destacada volatilidad presente en el mercado financiero durante los últimos años. De allí la importancia de incluir el componente de flexibilidad en las métricas tradicionales de valoración de proyectos.

Como logró identificarse en este trabajo, el sector de telecomunicaciones enfocado en la creación de un proyecto de video juegos, presenta alta aplicabilidad en los conceptos de opciones reales, debido a los componentes de incertidumbre que suponen este tipo de decisiones de inversión.

\section{REFERENCIAS}

Blazenko, G. W., Pavlov, A. D. y Eddy-Sumeke, F. (2012). New venture startups and technological innovation. International Journal of Managerial Finance, 8(1), 4-35. https://doi. org/10.1108/17439131211201013

Cassimon, D., Engelen, P. J. y Yordanov, V. (2011). Compound real option valuation with phase-specific volatility: A multi-phase mobile payments case study. Technovation, 31(5-6), 240 255. https://doi.org/10.1016/j.technovation. 2010.12 .004

Copeland, T. \& Antikarov, V. (2003). Real options: a practitioner's guide. Nueva York: Texere.

Della, M., Gryglewicz, S. y Kort, P. (2012). Optimal investment in learningcurve technologies. Journal of Economic Dynamics and Control, 36(10), 1462-1476. https://doi.org/10.1016/j. jedc.2012.03.014

Calle, A. y Tamayo, V. (2009). Decisiones de inversion a traves de opciones reales. Estudios Gerenciales, 25(111). 107-126. https://doi.org/10.1016/ S0123-5923(09)70073-7

Franklin, S. y Diallo, M. (2012). Valuing real options for network investment decisions and cost-based access pricing. Engineering Economist, 57(4), 223-246. https://doi.org/10.1080/001 3791X.2012.729874

González, L. (2008). Valoración de un proyecto de inversión a través de opciones reales para una empresa colombiana del sector de telecomunicaciones. Revista Soluciones de Postgrado EIA, 61-74.

Leippold, M. y Stromberg, J. (2017). Strategic technology adoption and hedging under incomplete markets. Journal of Banking and Finance, 81, 181-199. https://doi.org/10.1016/j. jbankfin.2016.09.008 
Martínez, F. y Aizenstat, A. (2006). Opciones reales, valuación financiera de proyectos y estrategias de negocios aplicaciones al caso Mexicano. Trimestre Economico, 73(2), 363-405. http:// dx.doi.org/10.20430/ete.v73i290.548

Mascareñas, J. (2008). Las decisiones de inversión como opciones reales: Un enfoque conceptual. [Documentos de Trabajo]. Madrid: Universidad Complutense. Recuperado de https:// eprints.ucm.es/6649/1/9805.pdf

Myers. (1977). Determinants of Corporate Borrowing. Journal of Financial Economics, 5(2). 147-175. https://doi. org/10.1016/0304-405X(77)90015-0

Rohner, P. (julio 14, 2018). Videojuegos, una industria silenciosa. La republica. [Actualidad]. Recuperado de https://www.larepublica.co/analisis/ pascal-rohner-515246/videojuegosuna-industria-silenciosa-2749242

Trigeorgis, L. y Ioulianou, S. (2013). Valuing a high-tech growth company: the case of EchoStar Communications Corporation. European Journal of Finance, 19(7-8), 734-759. https://doi.or g/10.1080/1351847X.2011.640343
Villani, G. (2014). Valuation of RyD Investment Opportunities with the Threat of Competitors Entry in Real Option Analysis. Computational Economics, 43(3), 331-355. https://doi.org/10.1007/s10614-0139370-2

Wadhwa, A. y Basu, S. (2013). Exploration and resource commitments in unequal partnerships: An examination of corporate venture capital investments. Journal of Product Innovation Management, 30(5), 916-936. https://doi.org/10.1111/jpim.12037

Wesseh, P. \& Lin, B. (2016). A real options valuation of Chinese wind energy technologies for power generation: Do benefits from the feedin tariffs outweigh costs? Journal of Cleaner Production, 112, 15911599. https://doi.org/10.1016/j.jclepro.2015.04.083 


\section{Biodata}

Armando Lenin Támara Ayús es Doctor en Administración con Magister en Finanzas y Especialista en diseño y evaluación de Proyectos. Profesor Titular de la Universidad EAFIT (Colombia). https:// orcid.org/0000-0003-3619-5164

Susana Marín Arango es Profesional en Finanzas de la Universidad EAFIT (Colombia). https://orcid.org/0000-00023466-1152

Santiago Cadavid Álvarez es Profesional en Finanzas de la Universidad EAFIT (Colombia). https://orcid.org/0000-00028579-940X 